\title{
Study of Dimer Lead Compounds via Triazole Rings as Novel Potential Antitumor Agents
}

\author{
Cheng Ting $\mathrm{Zi}^{1}$, Liu Yang ${ }^{2}$, Zi Hua Jiang ${ }^{3}$, Jiang Miao Hu*2, Xuan Jun Wang*1 and Jun Sheng*1 \\ ${ }^{1}$ Key Laboratory of Pu-er Tea Science, China \\ ${ }^{2}$ State Key Laboratory of Phytochemistry and Plant Resources in West China, China \\ ${ }^{3}$ Department of Chemistry, Canada \\ *Corresponding author: Jiang Miao Hu, State Key Laboratory of Phytochemistry and Plant Resources in West China, China \\ Xuan Jun Wang, Key Laboratory of Pu-er Tea Science, Ministry of Education, China \\ Jun Sheng, Key Laboratory of Pu-er Tea Science, Ministry of Education, China
}

\begin{tabular}{l} 
ARTICLE INFO \\
\hline Received: March 27, 2019 \\
Published: April 08, 2019 \\
Citation: Cheng Ting Zi, Liu Yang, Zi \\
Hua Jiang, Jiang Miao Hu, Xuan Jun \\
Wang, Jun Sheng. Study of Dimer Lead \\
Compounds via Triazole Rings as Nov- \\
el Potential Antitumor Agents. Biomed \\
J Sci \& Tech Res 16(5)-2019. BJSTR. \\
MS.ID.002915.
\end{tabular}

ABSTRACT

The $\mathrm{Cu}(\mathrm{I})$-catalyzed azide-alkyne cycloaddition (CuAAC) reaction was a powerful means for linking two molecules. The dimer lead compounds via triazole rings could promote the development of double target inhibitors, which might be an innovative strategy for potential antitumor agents, suggesting their potential as anticancer drugs for further development.

Keywords: CuAAC Reaction; 1,2,3-Triazole Ring; Lead Compound; Antitumor Agent

\section{Introduction}

Cancers still remain the second leading cause of death worldwide after cardiocascular diseases. It is estimated that about 18.1 million new cancer cases and 9.6 million cancer deaths in 2018 [1]. However, the medicines for treatment of malignant tumors are far from sufficient. So the development of novel agents with good therapeutic is the key topics which have been concerned currently [2]. Natural products based drug discovery has become a major strategy in modern pharmaceutical research [3], and roughly $60 \%$ of the currently used cancer chemotherapeutic agents are directly or indirectly derived from natural products, but the high toxicity, the lack of "true" tumor cell specificity, and the narrow therapeutic margins of the most natural compounds has limited their application as a drug in cancer chemotheraphy [4-7]. Recently, more attention has been attracted on targeting anti-cancer drugs: disrupting tumor-specific cell signaling, cell division, energy metabolism, gene expression, drug resistance and so on. The structural modification of natural compounds for developing new antitumor drugs with increased selectivity and reduced toxicity is highly desirable. For example, the 1,2,3-triazole ring is a widespread functional group in various drugs [8]. The $\mathrm{Cu}(\mathrm{I})$-catalyzed azide-alkyne cycloaddition (CuAAC) reaction (Click reaction) was a powerful means for linking two molecular fragments between a terminal alkyne and an azide to generate substituted 1,2,3-triazole (Figure 1) [9,10]. Click reaction has recently emerged as one of the most powerful tools in drug discovery, chemical biology, and proteomic applications.

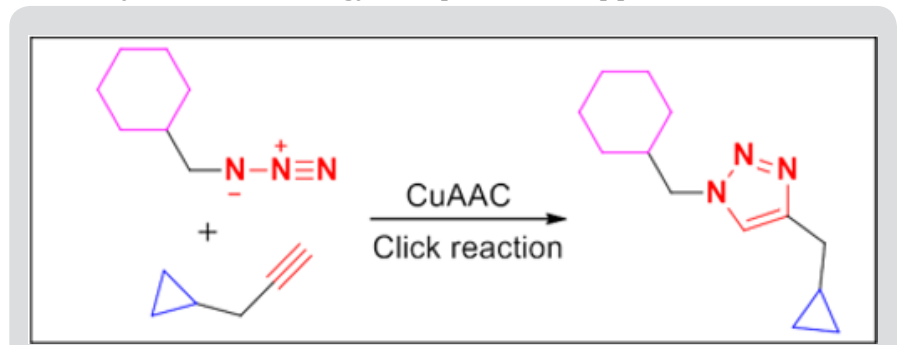

Figure 1: The $\mathrm{Cu}(\mathrm{I})$-catalyzed Husigen azide-alkyne 1,3-dipolar cycloaddition reaction (Click reaction). 
A dual inhibitor is likely to reduce the liabilities associated with combination treatments, particularly, off-target toxicities, drugdrug interactions, and additive effects [11]. In addition, dual target inhibitors would likely retain cytotoxic activity when resistance was acquired due to alteration of only one drug target. Thus, dual inhibitors are an attractive therapeutic approach in the drug development process. The Click reaction can link two molecular via 1,2,3-triazole ring, the strategy of dimer lead compounds via triazole rings as potential antitumor agents shown in Figure 2. As illustrated in Figure 2, two drugs (same or different drugs) linked via a triazole ring with the different length of the linking spacer between the drug and the 1,2,3-triazole (Figure 2I); or two drugs (same or different drugs) linked via two triazole rings with the different length of the linking spacer between the drug (1,2,3-triazole ring) and the 1,2,3-triazole (Figure 2 II).

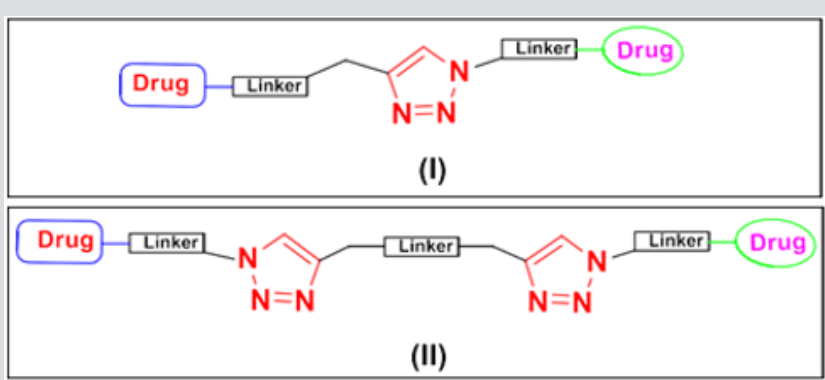

Figure 2: The strategy of dimer natural active lead compounds via triazole rings.

Recently, the Click reaction has been gaining popularity link two molecular fragment in creating a wide variety of drug-like molecules [8,12], such as paclitaxel [13], podophyllotoxin [14,15], and camptothecin [15]. Unpublished findings from our laboratory suggest that dimeric epigallocatechin gallate/ camptothecindervatives, and epigallocatechin gallate- $4 \beta$-triazolopodophyllotoxin/camptothecin conjugates showed highly potent anticancer activity. Thus, the strategy of dimer natural active lead compounds via triazole rings can promote the development of double target inhibitors which reduces the length and complexity of trials as well as costs.

\section{Conclusion}

$\mathrm{Cu}(\mathrm{I})$-catalyzed azide-alkyne cycloaddition (CuAAC) reaction was employed successfully to prepare a series triazole-linked derivatives. The available data so far produced suggest that the dimer lead compounds via triazole rings might be an innovative strategy for potential antitumor agents. It is extremely important to continue the discussion about the dimer lead compounds via triazole rings for development of cancer chemotherapeutic agents in further studies.

\section{Acknowledgment}

This work was supported by the National Nature Science Foundation of China for financial support (No. 21602196); the
Yunnan Provincial Science and Technology Department (Nos. 2017ZF003-04, 2017FD084 and 2017FG001-046); and Yunnan Agricultural University Natural Science Foundation for Young Scientists (No. 2015ZR08).

\section{References}

1. Bray F, Ferlay J, Soerjomataram I, Siegel RL, Torre LA, et al. (2018) Global cancer statistics 2018: GLOBOCAN estimates of incidence and mortality worldwide for 36 cancers in 185 countries. CA Cancer J Clin 68(6): 394424 .

2. Wen XS, Li D, Zhang YY, Liu SP, Ghali L, et al. (2012) Arsenic trioxide induces cervical cancer apoptosis, but specifically targets human papillomavirus-infected cell populations. Anti-cancer drugs 23(3): 280287.

3. Newman DJ, Cragg GM (2007) Natural products as sources of new drugs over the last 25 years. J Nat Prod 70(3): 461-477.

4. Nagle DG, Zhou YD, Mora FD, Mohammed KA, Kim Y (2004) Mechanism Targeted Discovery of Antitumor Marine Natural Products. Curr Med Chem 11(13): 1725-1756.

5. Berndsen RH, Abdul UK, Weiss A, Zoetemelk M, Te Winkel MT, et al. (2017) Epigenetic approach for angiostatic therapy: promising combinations for cancer treatment. Angiogenesis 20(2): 245-267.

6. Gabizon AA, Patil Y, La Beck NM (2016) New insights and evolving role of pegylated liposomal doxorubicin in cancer therapy. Drug Resist Updat 29: 90-106.

7. Sarkar S, Malekshah OM, Nomani A, Patel N, Hatefi A (2018) A novel chemotherapeutic protocol for peritoneal metastasis and inhibition of relapse in drug resistant ovarian cancer. Cancer Med 7(8): 3630-3641.

8. Krivopalov VP, Shkurko OP (2005) 1,2,3-Triazole and its derivatives. Development of methods for the formation of the triazole ring. Russ Chem Rev 74(4): 339-379.

9. Rostovtsev VV, Green LG, Fokin VV, Sharpless KB (2002) A stepwise Huisgen cycloaddition process: copper(I)-catalyzed regioselective "ligation" of azides and terminal alkynes. Angew Chem Int Ed 114: 27082711.

10. Tørnoe CW, Christensen C, Meldal M (2002) Peptidotriazoles on solid phase: [1,2,3]-triazoles by regiospecific copper(I)-catalyzed 1,3-dipolar cycloadditions of terminal alkynes to azide. J Org Chem 67(9): 30573064.

11. Liu S, Yosief HO, Dai L, Huang H, Dhawan G, et al. (2018) Structure-Guided Design and Development of Potent and Selective Dual Bromodomain 4 (BRD4)/Polo-like Kinase 1 (PLK1) Inhibitors. J Med Chem 61(17): 77857795.

12. Shafran EA, Bakulev VA, Rozin YA, Shafran YM (2008) Condensed 1,2,3-triazoles (review). Chem Heterocycl Compd 44(9): 1040-1069.

13. Pilkington Miksa M, Arosio D, Battistini L, Belvisi L, De Matteo M, et al. (2012) Design, synthesis, and biological evaluation of novel cRGDpaclitaxel conjugates for integrin-assisted drug delivery. Bioconjug Chem 23(8): 1610-1622.

14. Zi CT, Yang L, Xu FQ, Dong FW, Yang D, et al. (2018) Synthesis and anticancer activity of dimeric podophyllotoxin derivatives. Drug Des Dev Ther 12: 3393-3406.

15. Zi CT, Yang L, Dong FW, Kong QH, Ding ZT, et al. (2019) Synthesis and antitumor activity of camptothecin- $4 \beta$-triazolopodophyllotoxin conjugates. Nat Prod Res 12: 1-9. 
ISSN: 2574-1241

DOI: 10.26717/BJSTR.2019.16.002915

Jiang Miao H, Xuan Jun W, Jun S. Biomed J Sci \& Tech Res

(c) (P) This work is licensed under Creative

Submission Link: https://biomedres.us/submit-manuscript.php

$\begin{array}{ll}\text { BIOMEDICAL } & \text { Assets of Publishing with us } \\ \text { RESEARCHES } & \text { Global archiving of articles } \\ & \text { - Immediate, unrestricted online access } \\ \end{array}$

